\title{
Linearized min-max robust model predictive control: application to the control of a bioprocess
}

\author{
S. E. Benattia and S. Tebbani* ${ }^{\dagger}$ and D. Dumur \\ Laboratory of Signals and Systems (L2S, UMR CNRS 8506), CentraleSupélec-CNRS-Univ. Paris-Sud, Université \\ Paris-Saclay, France
}

\begin{abstract}
SUMMARY
This work deals with the problem of trajectory tracking for a nonlinear system with unknown but bounded model parameter uncertainties. First, this work focuses on the design of a robust nonlinear model predictive control (RNMPC) law subject to model parameter uncertainties implying solving a min-max optimization problem. Secondly, a new approach is proposed, consisting in relating the min-max problem to a more tractable optimization problem based on the use of linearization techniques, to ensure a good tradeoff between tracking accuracy and computation time. The developed strategy is applied in simulation to a simplified macroscopic continuous photobioreactor model and is compared to the RNMPC and nonlinear model predictive controllers. Its efficiency and its robustness against parameter uncertainties and/or perturbations are illustrated through numerical results. Copyright (c) 2016 John Wiley \& Sons, Ltd.
\end{abstract}

KEY WORDS: robust model predictive control; min-max problem; uncertain systems; stability; bioreactor

\section{INTRODUCTION}

The control of nonlinear uncertain systems subject to physical constraints on both input and state is undoubtedly a challenging and important issue, involving either stabilization or tracking problems. To cope with this challenge, well-known systematic nonlinear control methods such as feedback linearization and constructive Lyapunov-based methods $[1,2,3]$ lead to very elegant solutions, but they often rely on a complicated design procedure that does not scale well to large systems, and they cannot handle constraints easily or in a systematic manner. Based on this, the concept of optimal control and in particular the Receding Horizon Control (RHC) approach appears to be an attractive alternative since the complexity of the control design only increases moderately with the size and complexity of the system. Among RHC strategies, Model Predictive Control (MPC) is a powerful approach [4, 5], which allows the current control input to be determined,

${ }^{*}$ Correspondence to: Sihem Tebbani, Laboratory of Signals and Systems(L2S, UMR CNRS 8506), CentraleSupélecCNRS-Univ. Paris-Sud, Université Paris-Saclay, France

†E-mail: sihem.tebbani@centralesupelec.fr 
while taking account of the future system behavior and constraints on the system. This approach is based on the receding horizon principle, where a finite horizon open-loop optimal control problem is solved at each sampling instant, using a model of the system to forecast future process behavior over a given prediction horizon, and the optimized control trajectory is implemented until a new optimized control trajectory is available at the next sampling instant. For strongly nonlinear or uncertain systems, linear MPC may not be effective enough. In this case a nonlinear version can be implemented, the so-called Nonlinear Model Predictive Control (NMPC) [6, 7]. Nevertheless, including a nonlinear model within the MPC structure changes the problem from an online convex quadratic problem to a repeated online possibly non-convex nonlinear problem, which may become more difficult to solve [8]. In particular, special attention has to be given to the initial guess provided to the algorithm as an important parameter to ensure the convergence and the success of a given optimization problem.

Regardless of the linear or nonlinear formulation, the (N)MPC strategy is put forward because of its ability to deal with uncertainties and constraints. It has proven itself in several applications for many classes of systems and has had a significant impact on the world of industrial control engineering. Indeed, MPC is cited as one of the most popular advanced techniques for industrial process applications and has been widely adopted in the field of process control, due to the simplicity of the algorithm. As a consequence, there are many applications of predictive control strategies in very various domains, e.g. robot manipulators [9], clinical anesthesia [10], the cement industry and pulp factories [11], drying towers and robot arms [12], distillation columns [13, 14], Polyvinyl chloride (PVC) plants, steam generators and oil refining [15], solar power plants [6], thermomechanical pulping [16, 17], biochemical systems [18, 19, 20], motor control and food extruder processes [21], the petrochemical sector [22], aerospace [23, 24, 25], automotive [26], mining metallurgy [27, 28], etc.

However, even if NMPC strategies have proved to be efficient in many industrial applications thanks to their ability to operate the process safely under physical constraints, the monitoring of complex, highly nonlinear, uncertain systems becomes an increasingly delicate task. This is the case for example of bioprocesses, which will be the application field considered in this work. Indeed, in such cases, the size of the model parameters vector is usually important and model parameters are generally only known with a confidence interval (for example determined from an identification procedure). In this case, the performances of the NMPC law usually decrease when the true plant evolution deviates significantly from the one predicted by the model or when only a limited amount of data is available.

In this case, one solution is to move to an adequate robust control strategy in order to guarantee that the process will yield the reference trajectory under model parameter uncertainties. Therefore, NMPC strategies have to be extended to provide robustness features, developing robust control strategies, which can compensate for the lack of parameter information and/or accuracy, dealing at the same time with constraints and disturbances acting on the system. There are two popular alternatives for making decisions with incomplete knowledge: the stochastic solution (probabilistic theory) [29] and the min-max solution (game theory) [30, 31, 32, 33], which appear to be expensive approaches. Regarding the second solution, robust variants of NMPC (RNMPC) [34, 35] exist capable of taking account of set bounded disturbance, as they are formulated as a nonlinear min-max optimization problem. The effect of the uncertainties are taken into account in the design procedure, 
for which the objective function is minimized for the worst possible uncertainty realization. However, this approach tends to become too complex to be solved numerically online in the case of large scale systems. Consequently, the total calculation time is an important factor that must be reduced as much as possible.

The aim of this paper is thus to propose a new robust control law formulation that is computationally more tractable in calculating the optimal control compared to a min-max robust approach, which makes it suitable for online implementation, while ensuring a stable real time operation of the plant, close to a certain state or desired profile under model parameter uncertainties. Therefore, the proposed solution, referred to as Linearized Robust MPC (LRMPC), transforms the min-max RNMPC into a more tractable optimization problem, approaching the criterion through a model linearization technique (first order Taylor series expansion) at each sampling time along the nominal trajectory.

The paper is organized as follows. In Section 2, some notations used throughout the paper are introduced. Section 3 presents the class of nonlinear systems that will be considered. The RNMPC strategy, based on the min-max problem formulation, is firstly presented in section 4 . The robust predictive control strategy LRMPC, based on linearization techniques, is detailed section 5. An application to the control of biomass concentration in a continuous photobioreactor is presented in Section 6. Numerical results are provided in order to assess the effectiveness of the proposed strategy in the case of model mismatch. Finally, a conclusion and perspectives are presented in Section 7.

\section{NOTATIONS}

Let $\mathbb{N}, \mathbb{R}, \mathbb{R}_{\geq 0}$ and $\mathbb{Z}$ denote natural, real, non-negative real and integer number sets, respectively. $\mathbb{O}_{n \times m} \in \mathbb{R}^{n \times m}$ is the zero matrix of dimension $n \times m$ and $\square_{n} \in \mathbb{R}^{n \times n}$ is the identity matrix of dimension $n \times n$. The notation $A^{*}$ denotes the conjugate transpose of the matrix $A$. The notation $A^{\dagger}$ denotes the pseudo inverse of the matrix $A$ such that $A^{\dagger} \triangleq \lim _{\delta \rightarrow 0}\left(A^{*} A+\delta \llbracket\right)^{-1} A^{*}$. Given $n \in \mathbb{Z}_{\geq 0}$, an arbitrary norm of a vector $x \in \mathbb{R}^{n}$ is denoted as $|x| .\|z\|_{P}^{2}=z^{\top} P z$ is the Euclidean norm weighted by the matrix $P$. Matrix norm $\|A\|$ is given by $\|A\|=\sqrt{\bar{\sigma}\left(A^{*} A\right)}$ with $\bar{\sigma}(A)$ the maximum eigenvalue of A. A symmetric $n \times n$ real matrix $A$ is said to be positive semidefinite if the scalar $z^{\top} A z$ is nonnegative for every non-zero column vector $z$ of $n$ real numbers. It is denoted $A \succeq 0$. A symmetric $n \times n$ real matrix $A$ is said to be positive definite if the scalar $z^{\top} A z$ is positive for every non-zero column vector $z$ of $n$ real numbers. It is denoted $A \succ 0$. A matrix $A \in \mathbb{R}^{m \times n}$ is full column rank if and only if $A^{\top} A$ is invertible. Given an affine space $A$, a set $B \subseteq A$ is said to be convex if $\forall x, y \in B, \forall t \in[0,1]:(1-t) x+t y \in B$. Let $\mathrm{A}$ be a convex set and let $f: A \longrightarrow \mathbb{R}$. $f$ is said to be convex if $\forall a, b \in A, \forall t \in[0,1]: f(t a+(1-t) b) \leq t f(a)+(1-t) f(b)$. A function $f: \mathbb{R} \longrightarrow \mathbb{R}$ is said to be $\mathscr{C}^{n}$ function with $n \in \mathbb{N}$, if the first $n$ derivatives $f^{\prime}(),. f^{\prime \prime}(),. \ldots, f^{(n)}($.$) all exist and are$ continuous with respect to their argument. 


\section{MODEL FORMULATION AND CONTROL OBJECTIVE}

\subsection{Model formulation}

The first step to implement the predictive control strategy is achieved with the use of a prediction model. In this context, it is important to obtain a mathematical representation reproducing the behavior of the system to be controlled as accurately as possible. In our case, we will use a discrete formulation: discrete models for prediction and control.

Consider a system described by an uncertain continuous-time nonlinear model:

$$
\left\{\begin{array}{l}
\dot{x}(t)=F(x(t), u(t), \theta), x_{0}=\bar{x} \\
y(t)=H x(t)
\end{array}\right.
$$

where $x \in \mathbb{X} \subseteq \mathbb{R}^{n_{x}}$ is the state vector with $\mathcal{X}$ the compact set of admissible states. $\bar{x}$ is the initial state vector. $y \in \mathbb{\complement} \subseteq \mathbb{R}^{n_{y}}$ is the measured output with $\checkmark$ the compact set of admissible outputs. $u \in \mathbb{U} \subseteq \mathbb{R}^{n_{u}}$ represents the control input with $\mathbb{U}$ the compact set of admissible controls. $\theta \in \mathbb{R}^{n_{\theta}}$ is the vector of uncertain parameters that are assumed to lie in the compact set $\Theta=\left[\theta^{-}, \theta^{+}\right]$defined as follows:

$$
\theta=\theta_{\text {nom }}+\delta \theta
$$

where $\theta_{\text {nom }}$ is the nominal parameters vector defined as the average value (centroid) of the compact set:

$$
\theta_{\text {nom }}=\frac{\theta^{+}+\theta^{-}}{2}
$$

and $\delta \theta$ the parameter uncertainties vector. The mapping $F: \mathbb{R}^{n_{x}} \times \mathbb{R}^{n_{u}} \times \mathbb{R}^{n_{\theta}} \longrightarrow \mathbb{R}^{n_{x}}$, of class $\mathscr{C}^{2}$ with respect to all its arguments, represents the nonlinear process dynamics. The measurement matrix is given by $H \in \mathbb{R}^{n_{y} \times n_{x}}$.

\section{Remark 1}

The sets $\mathcal{X}$ and $\mathbb{U}$ are generally polyhedral convex sets, taking account, for example, of physical constraints acting on the system.

\section{Remark 2}

The measurement could also be nonlinear with respect to the state. It is assumed here linear to simplifiy mathematical developments.

Exogenous inputs can act on system (1). They are omitted to simplify the notation (but are applied to the system). Most models of real-life processes are given as continuous-time models, usually in the form of differential equations (1). A discrete formulation is used to convert these models for the purpose of controller design.

The discrete-time outputs are obtained at each constant sampling time $T_{s}$ by the integration of the continuous-time state space model (1) using, for example, the Runge-Kutta method with an integration time step $T_{d}$ (lower than the sampling time $T_{s}$ ). The control input $u(t)$ is parametrized using a piecewise-constant approximation over a time interval $\left.\left[t_{k}, t_{k+1}\right] \triangleq\left[k T_{s},(k+1) T_{s}\right)\right]$ : 
Let us define the discrete state trajectory $g$ as the solution, at time $t_{k+1}$, of system (1):

$$
\left\{\begin{array}{l}
x_{k+1}=g\left(t_{0}, t_{k+1}, \bar{x}, u_{t_{0}}^{t_{k}}, \theta\right) \\
y_{k}=H x_{k}
\end{array}\right.
$$

with initial state $\bar{x}=x_{0}$, and $u_{t_{0}}^{t_{k}}$ the control sequence from the initial time instant $t_{0}$ to the time instant $t_{k}$.

Thus, the prediction model could be defined by the following recursive equations:

$$
\left\{\begin{array}{l}
x_{k+1}=f\left(x_{k}, u_{k}, \theta\right), k \geq 0, x_{0}=\bar{x} \\
y_{k}=H x_{k}
\end{array}\right.
$$

where $x_{k+1}$ is the state at time $t_{k+1}, k$ is the time index, $x_{k}$ and $y_{k}$ are the discrete state vector and the sampled output at time $t_{k}$, respectively.

It can easily be shown that:

$$
f\left(x_{k}, u_{k}, \theta\right) \equiv g\left(t_{k}, t_{k+1}, x_{k}, u_{k}, \theta\right)
$$

In the sequel, model (5) will be used as the prediction model in the NMPC strategy (i.e. to predict the future behavior of the system).

\subsection{Control objectives}

In this study, the main objective is to force the output signal $y$ to follow a given reference trajectory $y^{r}$, while the control input $u$ is constrained to track a reference $u^{r}$. In addition, saturations on the state vector and control input signal with minimum and maximum thresholds $x_{\min }, x_{\max }, u_{\min }$ and $u_{\max }$ respectively can be included (defining the sets $\mathbb{X}$ and $\mathbb{U}$ as the constraints sets on the state and control signals respectively). These inequality constraints may result from both physical and operational constraints on the controlled system.

In this paper, the reference output $y^{r}$ is assumed to be determined from the application of the reference input $u^{r}$ to the system, using prediction model (5). More generally, the tracking problem is assumed to be feasible.

\section{RNMPC STRATEGY AS A MIN-MAX OPTIMIZATION PROBLEM}

As mentioned in section 1, the NMPC problem is mainly formulated for trajectory tracking purposes. The predictive controller predicts the future evolution of the plant over a finite time receding horizon, using a nonlinear dynamic model. At each time instant, the optimal control sequence over the prediction horizon is computed by minimizing a quadratic criterion expressed as the weighted sum of future output and tracking errors and future control tracking errors, while ensuring that all constraints are respected. The first control in the optimal sequence is applied to the system until the next time step, when the measurement becomes available. The optimization problem is solved again at the next sampling time according to the well-known receding horizon principle [6, 7]. 
Since the predictive controller is model-based, it is very sensitive to model uncertainties and, more specifically, to the model parameter values. In this context, it will be assumed hereafter that the parameter vector $\theta$ is uncertain and belongs to a known set $\Theta$ as stated in section 3.1. Thus, robustification in the presence of model uncertainties naturally leads to the formulation of a nonlinear min-max optimization problem $[43,44,33]$. The min-max RNMPC strategy is recalled in this section.

Considering the RNMPC formulation, the control sequence that minimizes a worst case cost function is derived from the following optimization problem (at time index $k$ ):

$$
{\stackrel{\star}{\mathfrak{k}_{k}}}^{k+N_{p}-1}=\arg \min _{\substack{k+N_{p}-1 \\ u_{k}}} \max _{\delta \theta} \Pi_{\mathrm{RNMPC}}\left(x_{k}, u_{k}^{k+N_{p}-1}, \delta \theta\right)
$$

subject to the constraints in a matrix form:

$$
\left\{\begin{array}{l}
\hat{x}_{k+j}=g\left(t_{k}, t_{k+j}, x_{k}, u_{k}^{k+j-1}, \theta=\theta_{\text {nom }}+\delta \theta\right), j=\overline{1, N_{p}} \\
\theta \in \Theta \\
{\left[\begin{array}{cc}
\mathbb{\square}_{n_{x}} & \mathbb{O}_{n_{u}} \\
-\square_{n_{x}} & \mathbb{O}_{n_{u}} \\
\mathbb{O}_{n_{x}} & \mathbb{\square}_{n_{u}} \\
\mathbb{O}_{n_{x}} & -\mathbb{\square}_{n_{u}}
\end{array}\right]\left[\begin{array}{c}
\hat{x}_{k+j} \\
u_{k+j-1}
\end{array}\right] \leq\left[\begin{array}{c}
x_{\max } \\
-x_{\min } \\
u_{\max } \\
-u_{\min }
\end{array}\right], \forall j=\overline{1, N_{p}}}
\end{array}\right.
$$

where $\theta$ and $\theta_{\text {nom }}$ are given in (2) and (3), $N_{p}$ is the length of the prediction horizon.

The cost function that will be minimized is expressed as the sum of two quadratic functions based on tracking errors over the receding horizon and defined as (at time $t_{k}$ ):

$$
\Pi_{\mathrm{RNMPC}}\left(x_{k}, u_{k}^{k+N_{p}-1}, \delta \theta\right) \triangleq\left\|u_{k}^{k+N_{p}-1}-u_{k}^{r, k+N_{p}-1}\right\|_{V}^{2}+\left\|\hat{y}_{k+1}^{k+N_{p}}-y_{k+1}^{r, k+N_{p}}\right\|_{W}^{2}
$$

with $x_{k}$ the state vector at time $t_{k}$,

$u_{k}^{k+N_{p}-1}=\left[\begin{array}{c}u_{k} \\ \vdots \\ u_{k+N_{p}-1}\end{array}\right]$ the optimization vector, $u_{k}^{r, k+N_{p}-1}=\left[\begin{array}{c}u_{k}^{r} \\ \vdots \\ u_{k+N_{p}-1}^{r}\end{array}\right]$ the reference control sequence, the predicted outputs $\hat{y}_{k+1}^{k+N_{p}}$ are defined as:

$$
\hat{y}_{k+1}^{k+N_{p}}=\left[\begin{array}{c}
H g\left(t_{k}, t_{k+1}, x_{k}, u_{k}, \theta\right) \\
H g\left(t_{k}, t_{k+2}, x_{k}, u_{k}^{k+1}, \theta\right) \\
\vdots \\
H g\left(t_{k}, t_{k+N_{p}}, x_{k}, u_{k}^{k+N_{p}-1}, \theta\right)
\end{array}\right]
$$

and $y_{k+1}^{r, k+N_{p}}=\left[\begin{array}{c}y_{k+1}^{r} \\ \vdots \\ y_{k+N_{p}}^{r}\end{array}\right]$ represents the setpoint values.

where the subscript is related to the time instant.

$V \succ 0$ and $W \succ 0$ are tuning weighting matrices. 
The optimal control sequence $\star_{k}^{k+N_{p}-1}$ is determined to minimize the output and control tracking errors by considering all trajectories over all possible data scenarii $[45,46]$.

It is assumed that the tracking problem remains feasible for all realizations of the parameter values. In other words, it is assumed that there exists a feasible control input sequence for every set of parameter values within the known set $\Theta$.

\section{Remark 3}

For the min-max problem (8), at sampling time $(k+1) T_{s}$ the optimization variables $u_{k+1}^{k+N_{p}}$ and $\delta \theta$ are initialized by the optimal control sequence ${\stackrel{\star}{k} k+N_{p}-1}_{k+1}$ and the optimal parameter vector $\delta \stackrel{\star}{\theta}$ obtained from the optimization (8) at time instant $k$ as follows:

$$
\begin{aligned}
& \left(u_{k+1}^{k+N_{p}}\right)_{\mathrm{ini}}=\left[\stackrel{\star \star ⿱ ⺌}{k}^{k+N_{p}-1}, \stackrel{\star}{u}_{k+N_{p}-1}\right], \quad \forall k \geq 0 \\
& \delta \theta_{\text {ini }}=\delta \stackrel{\star}{\theta}=\arg \max _{\delta \theta} \Pi_{\mathrm{RNMPC}}\left(x_{k}, \stackrel{\star}{u}_{k}^{k+N_{p}-1}, \delta \theta\right)
\end{aligned}
$$

It clearly appears that the computation load grows with the size of the parameter vector, the number of control inputs and the prediction horizon, while the control strategy has to be implemented online. The challenge is thus to reduce the computation burden while maintaining good performance in term of accuracy. This motivates the elaboration of a new control robust predictive strategy with a limited time computation.

\section{LINEARIZED ROBUST MPC}

Since the min-max optimization problem (8)-(11) is time consuming, it will subsequently be replaced by a more tractable optimization problem, reducing the computational burden of the initial problem. The key idea is to approach the predicted outputs based on the nonlinear model through a linearization technique, around the reference control inputs and the nominal model parameters.

As a direct result of the linearization, the non-convex problem will be transformed into a convex one, for which more flexible tools will be accessible to handle the optimization problem more effectively.

\subsection{LRMPC formulation}

In the following approach, the outputs in the moving time frame are predicted by Taylor series expansion. A similar dual problem for robust state estimation, consisting in the design of a recedinghorizon observer, was presented in [47]. In this section, we propose to adapt this approach to the case of NMPC law design.

Based on a first order Taylor series expansion of (7), the prediction model $g$ for time $t_{k+1}$, starting from state $x_{k}$, is linearized around the reference control $u_{k}^{r}$ and for the nominal parameters $\theta_{\text {nom: }}$ :

$$
\hat{x}_{k+1} \approx \hat{x}_{\mathrm{nom}, k+1}+\nabla_{\theta} g\left(t_{k+1}\right) \delta \theta+\nabla_{u} g\left(t_{k+1}\right)\left(u_{k}-u_{k}^{r}\right) \triangleq f_{p}\left(x_{k}, u_{k}, \theta_{\mathrm{nom}}+\delta \theta\right)
$$


with

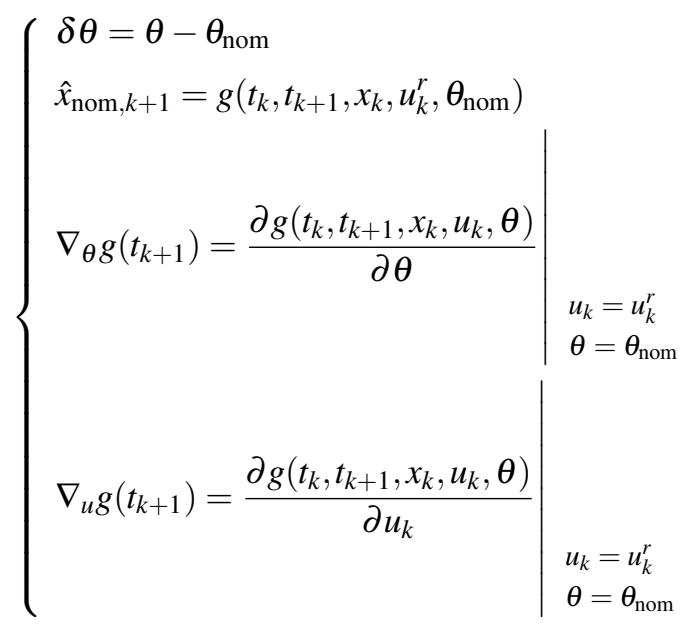

The model is still nonlinear with respect to the state vector (given by (14)). Only the effects of control input and variations in parameter values are considered for the linearization approach.

Generalizing, the predicted state for time $t_{k+j}$, starting from the state at $t_{k}$, is linearized around the control sequence $u_{k}^{r, k+j-1}$ and for $\theta_{\text {nom. }}$. Using the same approach as in (12) for $j=\overline{1, N_{p}}$, we obtain the following:

$$
\hat{x}_{k+j} \approx \hat{x}_{\mathrm{nom}, k+j}+\nabla_{\theta} g\left(t_{k+j}\right) \delta \theta+\nabla_{u} g\left(t_{k+j}\right)\left(u_{k}^{k+j-1}-u_{k}^{r, k+j-1}\right)
$$

with

$$
\left\{\begin{array}{l|l}
\hat{x}_{\mathrm{nom}, k+j}=g\left(t_{k}, t_{k+j}, x_{k}, u_{k}^{r, k+j-1}, \theta_{\mathrm{nom}}\right) & \\
\nabla_{\theta} g\left(t_{k+j}\right)=\frac{\partial g\left(t_{k}, t_{k+j}, x_{k}, u_{k}^{k+j-1}, \theta\right)}{\partial \theta} & \begin{array}{l}
u_{k}^{k+j-1}=u_{k}^{r, k+j-1} \\
\theta=\theta_{\text {nom }}
\end{array} \\
\nabla_{u} g\left(t_{k+j}\right)=\frac{\partial g\left(t_{k}, t_{k+j}, x_{k}, u_{k}^{k+j-1}, \theta\right)}{\partial u_{k}^{k+j-1}} & \begin{array}{l}
u_{k}^{k+j-1}=u_{k}^{r, k+j-1} \\
\theta=\theta_{\mathrm{nom}}
\end{array}
\end{array}\right.
$$

Different approaches are possible for determining the sensitivity functions defined in (19) and (20) with respect to the parameter vector and the control sequence respectively.

The dynamics of the sensitivity function with respect to $\theta$ can be computed for time $t \in\left[t_{k}, t_{k+N_{p}}\right]$ by solving numerically the following differential equation (from (1)):

$$
\frac{d}{d t}\left(\nabla_{\theta} g(t)\right)=\frac{\partial F\left(x(t), u(t), \theta_{\mathrm{nom}}\right)}{\partial x} \nabla_{\theta} g(t)+\left.\frac{\partial F(x(t), u(t), \theta)}{\partial \theta}\right|_{\theta=\theta_{\mathrm{nom}}}
$$


with as an initial condition:

$$
\nabla_{\theta} g\left(t_{k}\right)=\mathbb{O}_{n_{x} \times n_{\theta}}
$$

An alternative procedure is to use the finite differences in order to approximate numerically the derivatives $\nabla_{\theta} g$ for each parameter $\theta_{l}, l \in\left[1, n_{\theta}\right]$ and $\nabla_{u} g\left(t_{k+j}\right)$ for each control $u_{j}, j \in$ $\left[k, k+N_{p}-1\right]$.

The finite difference method approximates the $(i, j)$-th element of the Jacobian of a vector function $g(z)$ as

$$
\nabla g(z) \approx \frac{g_{i}\left(z_{j}+\delta\right)-g_{i}\left(z_{j}\right)}{\delta}
$$

for some small $\delta>0$. A too large $\delta$ will induce inaccuracies due to the nonlinearity of $g_{i}$, since the method computes the average slope between two points.

\section{Remark 4}

The most accurate result and computationally most efficient approach is to calculate gradients analytically (by symbolic differentiation). Doing this by hand, or even using symbolic computations, may quickly become intractable for MPC problems that may contain a large number of variables and parameters.

In order to simplify the calculation of the gradients $\nabla_{\theta} g$ and $\nabla_{u} g$, finite differences are used below to approximate numerically the derivatives $\nabla_{\theta} g\left(t_{k+j}\right)$ and $\nabla_{u} g\left(t_{k+i}\right)$.

From (11) and (17), the predicted ouputs over the moving horizon are expressed as follows:

$$
\hat{y}_{k+1}^{k+N_{p}}=G_{\mathrm{nom}, k+1}^{k+N_{p}}+G_{\theta, k+1}^{k+N_{p}} \delta \theta+G_{u, k}^{k+N_{p}-1}\left(u_{k}^{k+N_{p}-1}-u_{k}^{r, k+N_{p}-1}\right)
$$

where

$G_{\mathrm{nom}, k+1}^{k+N_{p}}=\left[\begin{array}{c}H \hat{x}_{\mathrm{nom}, k+1} \\ \vdots \\ H \hat{x}_{\mathrm{nom}, k+j} \\ \vdots \\ H \hat{x}_{\mathrm{nom}, k+N_{p}}\end{array}\right]$, is the column vector containing the predicted output for the nominal

case.

$G_{\theta, k+1}^{k+N_{p}}=\left[\begin{array}{c}H \nabla_{\theta} g\left(t_{k+1}\right) \\ \vdots \\ H \nabla_{\theta} g\left(t_{k+j}\right) \\ \vdots \\ H \nabla_{\theta} g\left(t_{k+N_{p}}\right)\end{array}\right]$, regroups the Jacobian matrices related to the parameters.

$G_{u, k}^{k+N_{p}-1}=\left[\begin{array}{c}H \nabla_{u} g\left(t_{k+1}\right) \\ \vdots \\ H \nabla_{u} g\left(t_{k+j}\right) \\ \vdots \\ H \nabla_{u} g\left(t_{k+N p}\right)\end{array}\right]$, regroups the Jacobian matrices related to the control sequence.

Assuming that the uncertain parameters are uncorrelated and recalling that

$$
\theta^{-} \leq \theta \leq \theta^{+}
$$


and

$$
\theta_{\text {nom }}=\frac{\theta^{+}+\theta^{-}}{2}
$$

Thus,

$$
\frac{\theta^{-}-\theta^{+}}{2} \leq \theta-\theta_{\text {nom }} \leq \frac{\theta^{+}-\theta^{-}}{2}
$$

Then, the bounded parametric error $\delta \theta$ can be expressed by:

$$
\delta \theta=\gamma \delta \theta_{\max }
$$

with

$$
\delta \theta_{\max }=\left(\theta^{+}-\theta^{-}\right) / 2
$$

and

$$
\|\gamma\| \leq 1
$$

The initial objective function $\Pi_{\text {RNMPC }}$ (10) is substituted by a cost function using the equation (24). The result is given by the following expression:

$$
\begin{aligned}
& \Pi_{\mathrm{RNMPC}}\left(x_{k}, u_{k}^{k+N_{p}-1}, \delta \theta\right) \approx\left\|u_{k}^{k+N_{p}-1}-u_{k}^{r, k+N_{p}-1}\right\|_{V}^{2}+ \\
& \left\|G_{\text {nom }, k+1}^{k+N_{p}}-y_{k+1}^{r, k+N_{p}}+G_{\theta, k+1}^{k+N_{p}} \delta \theta+G_{u, k}^{k+N_{p}-1}\left(u_{k}^{k+N_{p}-1}-u_{k}^{r, k+N_{p}-1}\right)\right\|_{W}^{2} \\
& \triangleq \Pi\left(x_{k}, u_{k}^{k+N_{p}-1}, \delta \theta\right)
\end{aligned}
$$

The new optimization problem is given by:

$$
\stackrel{\star}{u}_{k}^{k+N_{p}-1}=\arg \min _{\substack{k+N_{p}-1 \\ u_{k}}} \max _{\delta \theta} \Pi\left(x_{k}, u_{k}^{k+N_{p}-1}, \delta \theta\right)
$$

subject to

$$
\left\{\begin{array}{l}
\theta \in \Theta, x \in \mathbb{X}, u \in \mathbb{U} \\
\delta \theta=\gamma \delta \theta_{\max },\|\gamma\| \leq 1
\end{array}\right.
$$

Stability properties of the robust model predictive control strategy taking account of bounded uncertainties have been analyzed in [36, 37, 38, 39, 40, 41, 42]. The robust stability of the closedloop system (6) with (32)-(33) can be analyzed by exploiting the results obtained in [41, 38, 35]. An ongoing study is considering the stability analysis of the proposed control strategy.

\subsection{Calculation of the control sequence}

The optimization problem (32)-(33) is solved by means of a robust regularized least squares strategy in the presence of uncertain data, following an approach developed by Sayed et al. [48]. This approach is thus dedicated to the case of bounded constraints on the control inputs and there are no constraints on the state vector.

Let us consider the following robust regularized least squares (RRLS) problem:

$$
\min _{\underline{z} \leq z \leq \bar{z}} \max _{\delta A, \delta b}\left[\|z\|_{V}^{2}+\|(A+\delta A) z-(b+\delta b)\|_{W}^{2}\right]
$$


where

- $A$ is a known $m \times n$ matrix,

- $z$ is an unknown $n$-dimensional column vector,

- $b$ is a known $m \times 1$ vector,

- $W \succ 0$ and $V \succ 0$ are positive-definite weighting matrices.

The uncertainties $\delta A \in \mathbb{R}^{m \times n}$ and $\delta b \in \mathbb{R}^{m}$ can be structured under the following factored form:

$$
\left\{\begin{array}{l}
\delta A=C \Delta E_{a} \\
\delta b=C \Delta E_{b}
\end{array}\right.
$$

where $\Delta$ denotes an arbitrary contraction term with $\|\Delta\| \leq 1$, with a known matrix $C \in \mathbb{R}^{m \times n_{\xi}}$ not identically null and where $E_{a}$ and $E_{b}$ are known quantities of appropriate dimensions.

In the sequel, the uncertainties $\delta A$ and $\delta b$ are replaced by a perturbation vector $\xi \in \mathbb{R}^{n \xi}$ which is assumed to satisfy the following factored form:

$$
C \xi=\delta A z-\delta b=C \Delta\left(E_{a} z-E_{b}\right)
$$

Since $\|\Delta\| \leq 1, \xi$ is therefore constrained as follows:

$$
\|\xi\| \leq\left\|E_{a} z-E_{b}\right\| \triangleq \Gamma(z)
$$

The non-negative function $\Gamma(z)$ is assumed to be a known bound on the perturbation $\xi$ and is only a function of $z$.

With (37) and (38), the optimization problem (34) can be expressed as follows:

$$
\min _{\underline{z} \leq z \leq \bar{z}} \max _{\|\xi\| \leq \Gamma(z)}\left[\|z\|_{V}^{2}+\|A z-b+C \xi\|_{W}^{2}\right]
$$

The maximization subproblem is transformed into a standard form, which will further make it possible to define the corresponding Lagrange dual problem:

$$
\min _{z \leq \leq \leq \bar{z}} \min _{\|\xi\| \leq \Gamma(z)}\left[-\|z\|_{V}^{2}-\|A z-b+C \xi\|_{W}^{2}\right]
$$

The constrained subproblem on $\xi$ is solved by considering the Lagrangian duality [49]. We define the Lagrangian $L: \mathbb{R}^{n} \times \mathbb{R}^{n_{\xi}} \times \mathbb{R}^{+} \longrightarrow \mathbb{R}$ associated with the optimization problem (40) as

$$
L(z, \xi, \lambda) \triangleq-\|z\|_{V}^{2}-\|A z-b+C \xi\|_{W}^{2}+\lambda\left(\|\xi\|^{2}-\Gamma(z)^{2}\right)
$$

where $\lambda$ is the Lagrange multiplier associated with the inequality constraint (38) on $\xi$. Consequently, the problem (40) becomes equivalent to

$$
\min _{\underline{z} \leq z \leq \bar{z}} \max _{\lambda \geq 0} \min _{\xi} L(z, \xi, \lambda)
$$

Since $L(z, \xi, \lambda)$ is a convex quadratic function of $\xi$, we can find an explicit solution of $\xi$ which depends on the two variables $z$ and $\lambda$ by cancelling the gradient of the Lagrangian with respect to 
$\xi$, leading to:

$$
\xi^{\star}(z, \lambda)=\left(\lambda \rrbracket-C^{\top} W C\right)^{\dagger} C^{\top} W(A z-b)
$$

where $\mathbb{1}$ is the identity matrix with an appropriate dimension.

Due to the fact that the Hessian of the Lagrangian function (41) with respect to $\xi$ must be nonnegative at the optimum:

$$
\frac{\partial^{2} L}{\partial \xi^{2}}=-C^{\top} W C+\lambda \llbracket \succeq 0
$$

It turns out that the dual variable $\lambda$ must satisfy the following inequality constraint

$$
\lambda \geq\left\|C^{\top} W C\right\|
$$

Thanks to (43) and (45), problem (42) becomes

$$
\min _{\underline{z} \leq z \leq \bar{z}} \max _{\lambda \geq\left\|C^{\top} W C\right\|} L(z, \lambda)
$$

with

$$
L(z, \lambda)=-\|z\|_{V}^{2}-\|A z-b\|_{W(\lambda)}^{2}-\lambda \Gamma(z)^{2}
$$

in which the modified weighting matrix $W(\lambda)$ is derived from $W$ via:

$$
W(\lambda)=W+W C\left(\lambda \rrbracket-C^{\top} W C\right)^{\dagger} C^{\top} W
$$

The optimization problem (46) is further replaced by:

$$
\min _{\underline{z} \leq z \leq \bar{z}} \min _{\lambda \geq\left\|C^{\top} W C\right\|} \mathbb{J}(z, \lambda) \Longleftrightarrow \min _{\lambda \geq\left\|C^{\top} W C\right\|} \min _{z \leq z \leq \bar{z}} \mathbb{J}(z, \lambda)
$$

where the cost function $\rrbracket(z, \lambda)$ is defined as follows

$$
\mathbb{J}(z, \lambda) \triangleq\|z\|_{V}^{2}+\|A z-b\|_{W(\lambda)}^{2}+\lambda \Gamma(z)^{2}
$$

From (38), the optimization problem (49) is rewritten as:

$$
\min _{\lambda \geq\left\|C^{\top} W C\right\|} \min _{z \leq z \leq \bar{z}}\left[\|z\|_{V}^{2}+\lambda\left\|E_{a} z-E_{b}\right\|^{2}+\|A z-b\|_{W(\lambda)}^{2}\right]
$$

The corresponding formulation of the optimization problem (51) into a bilevel optimization problem can be written as follows:

$$
\begin{aligned}
\lambda^{\star}= & \arg \min _{\lambda \geq\left\|C^{\top} W C\right\|} \mathbb{J}(z(\lambda), \lambda) \\
& \text { s.t. } z(\lambda)=\arg \min _{\underline{z} \leq z \leq \bar{z}} \mathbb{l}(z, \lambda)
\end{aligned}
$$

The problem (52) is therefore structured as a two-level optimization problem: 


\section{- Lower-level}

The minimum $z(\lambda)$ is the solution of the following quadratic programming problem:

$$
\begin{aligned}
& \min _{z} \frac{1}{2} z^{\top} \mathscr{H} z+\mathscr{F}^{\top} z \\
& \text { subject to }\left[\begin{array}{cc}
\square & 0 \\
0 & -\square
\end{array}\right] z \leq\left[\begin{array}{c}
\bar{z} \\
-\underline{z}
\end{array}\right]
\end{aligned}
$$

with

$$
\left\{\begin{array}{l}
\mathscr{H}=2\left(V(\lambda)+A^{\top} W(\lambda) A\right) \\
\mathscr{F}=-2\left(A^{\top} W(\lambda) b+\lambda E_{a}^{\top} E_{b}\right)
\end{array}\right.
$$

where $W(\lambda)$ is given by (48) and the modified weighting matrix $V(\lambda)$ is obtained from $V$ via:

$$
V(\lambda)=V+\lambda E_{a}^{\top} E_{a}
$$

\section{- Upper-level}

The non-negative scalar parameter $\lambda^{\star} \in \mathbb{R}^{+}$is computed from the following unidimensional minimization problem:

$$
\lambda^{\star}=\arg \min _{\lambda \geq\left\|C^{\top} W C\right\|}\left[\|z(\lambda)\|_{V}^{2}+\lambda\left\|E_{a} z(\lambda)-E_{b}\right\|^{2}+\|A z(\lambda)-b\|_{W(\lambda)}^{2}\right]
$$

Finally, the bilevel problem (52) has a unique global minimum $\stackrel{\star}{z}$ given by (53) for $\lambda=\lambda^{\star}$ (i.e. $\left.\stackrel{\star}{z}=z\left(\lambda^{\star}\right)\right)$.

Based on this formalism, the min-max optimization problem defined by problem (32)-(33) with criterion (31), is reformulated under the form (34)-(38) with:

$$
\min _{\underline{z} \leq z \leq \bar{z}} \max _{\|\xi\| \leq\left\|E_{a} z-E_{b}\right\|}\left[\|z\|_{V}^{2}+\|A z-b+C \xi\|_{W}^{2}\right]
$$

and

$$
\left\{\begin{array}{l}
z=u_{k}^{k+N_{p}-1}-u_{k}^{r, k+N_{p}-1} \\
A=G_{u, k}^{k+N_{p}-1} \\
b=y_{k+N_{p}}^{r+1}-G_{\text {nom }, k+1}^{k+N_{p}} \\
C=G_{\theta, k+1}^{k+N_{p}} \\
\Delta=\gamma, E_{a}=0, E_{b}=-\delta \theta_{\max }
\end{array}\right.
$$

The application of (53)-(56) provides the solution of (32)-(33) with criterion (31) and inequality constraints on $u_{k}^{k+N_{p}-1}$ as follows:

Step 1. The scalar $\lambda^{\star}$ is computed from the following minimization problem:

$$
\lambda^{\star}=\arg \min _{\lambda \geq\left\|G_{\theta, k+1}^{k+N_{p}^{\top}} W G_{\theta, k+1}^{k+N_{p}}\right\|} \rrbracket(z(\lambda), \lambda)
$$


where the function $\mathbb{J}(z(\lambda), \lambda)$ is defined by:

$$
\mathbb{J}(z(\lambda), \lambda)=\|z(\lambda)\|_{V}^{2}+\lambda\left\|\delta \theta_{\max }\right\|^{2}+\left\|G_{u, k}^{k+N_{p}-1} z(\lambda)-y_{k+1}^{r, k+N_{p}}+G_{\mathrm{nom}, k+1}^{k+N_{p}}\right\|_{W(\lambda)}^{2}
$$

and $z(\lambda)$ is given by

$$
z(\lambda)=\arg \min _{\underline{z} \leq z \leq \bar{z}}\left[z^{\top} E(\lambda) z-2 B(\lambda)^{\top} z\right]
$$

where

$$
\left\{\begin{array}{l}
E(\lambda)=V+G_{u, k}^{k+N_{p}-1^{\top}} W(\lambda) G_{u, k}^{k+N_{p}-1} \\
B(\lambda)=G_{u, k}^{k+N_{p}-1^{\top}} W(\lambda)\left(y_{k+1}^{r, k+N_{p}}-G_{\mathrm{nom}, k+1}^{k+N_{p}}\right) \\
\underline{z}=u_{\min } \rrbracket_{n_{u}}-u_{k}^{r, k+N_{p}-1} \\
\bar{z}=u_{\max } \rrbracket_{n_{u}}-u_{k}^{r, k+N_{p}-1}
\end{array}\right.
$$

with $W(\lambda)$ given by:

$$
W(\lambda)=W+W G_{\theta, k+1}^{k+N_{p}}\left(\lambda \rrbracket-G_{\theta, k+1}^{k+N_{p}^{\top}} W G_{\theta, k+1}^{k+N_{p}}\right)^{\dagger} G_{\theta, k+1}^{k+N_{p}^{\top}} W
$$

Step 2. The control sequence $\stackrel{\star}{k}_{k}^{k+N_{p}-1}$ is derived from (61) for $\lambda=\lambda^{\star}$ :

$$
{\stackrel{\star}{u_{k}}}^{k+N_{p}-1}=u_{k}^{r, k+N_{p}-1}+z\left(\lambda^{\star}\right)
$$

To summarize, the predictive controller consists in solving online a bilevel optimization problem (52) instead of solving a min-max problem (8-10): a quadratic programming problem (53) in the lower level, and a unidimensional optimization problem (56) in the upper level. Since there are very efficient algorithms for this kind of optimization problems and that the two problems are convex, the obtained optimization problem remains more tractable than the min-max problem. In the sequel, this predictive control law will be referred to as Linearized Robust Model Predictive Controller (LRMPC). The LRMPC algorithm is summarized hereafter.

\section{LRMPC Algorithm}

\section{Inputs:}

$T_{s}$ : sampling time,

$T_{d}$ : integration time step,

$y^{r}, u^{r}$ : reference outputs and control inputs, respectively,

$x_{0}$ : initial state vector,

$\theta_{\text {nom }}:$ nominal parameters,

$\delta \theta_{\max }$ : maximum parameter uncertainties,

$N_{p}$ : length of the prediction horizon,

$W, V$ : weighting matrices on the outputs and the control inputs, respectively. 


\section{Outputs:}

1. Initialization: $k=1$

2. Update $x_{k}, y_{k+1}^{r, k+N_{p}}, \stackrel{\star^{k+N_{p}-2}}{k-1}$

3. Compute $G_{\text {nom }, k}^{k+N_{p}-1}, G_{\theta, k}^{k+N_{p}-1}, G_{u, k}^{k+N_{p}-1}$ according to equations (18-20)

4. Solve the bilevel optimization problem (52)

4.1. Optimize $\lambda^{\star}$ by solving the unidimensional problem (59), by solving QP problem (61) for each $\lambda$

4.2. Optimize $\stackrel{\star}{u}_{k}^{k+N_{p}-1}$ by solving the QP problem (61) for $\lambda=\lambda^{\star}$

5. Apply $\stackrel{\star}{u_{k}}$ to the system

6. Save $x_{k+1}$

7. $k \longleftarrow k+1$

8. return to 2

It should be mentioned that the LRMPC approach can be used in the case of more complex constraints on the control input (not only bounds). In this case, problem (61) becomes an NLP problem with a quadratic cost function and nonlinear constraints. It can be solved with an SQP algorithm for example.

In addition, this algorithm and formulation do not explicitly take account of the state constraints (here bounds on the state vector). The assumption that the solution of the tracking problem exists for all parameter realizations will make it possible to respect this assumption in general and the proposed algorithm could be used. The state constraints could also be taken into account by adding nonlinear constraints to the problem (61) (using model prediction). The problem to be solved then becomes an NLP problem with a quadratic cost function and nonlinear constraints.

\section{APPLICATION TO A BIOPROCESS}

In this section, the proposed approaches developed in the previous section are applied to the control of microalgae culture in a photobioreactor through a specific case study: the cultivation of I. galbana in a continuous photobioreactor [50]. A Droop model is used to describe the internal nutrient quantity per unit of biomass evolution. The aim here is to control biomass concentration in the photobioreactor through the dilution rate. The performance of the proposed control strategy will be compared to a nonlinear predictive controller and a min-max robust predictive controller. The idea is to highlight the performance of the LRNMPC strategy in comparison with similar control strategies.

\subsection{Model description}

The specificity of microalgae is that inorganic substrate uptake and growth are decoupled thanks to an intracellular storage of nutrients [51]. In order to take into account this phenomenon, the growth of microalgae is represented by a Droop model $[52,51]$ which decouples growth from substrate uptake, leading to the definition of an internal cell quota (i.e., the internal nutrient quantity per unit of biomass), and describes the growth rate as a function of the internal quota only. The mass 
balance model involves three state variables: the biomass concentration (denoted $X$, in $\mu \mathrm{m}^{3} \mathrm{~L}^{-1}$ ), the internal quota (denoted $Q$, in $\mu \mathrm{mol} \mu \mathrm{m}^{-3}$ ), and the substrate concentration (denoted $S$, in $\mu \mathrm{mol} \mathrm{L}{ }^{-1}$ ). The considered dynamic model assumes that the photobioreactor is in continuous mode (medium withdrawal flow rate equals its supply rate, leading to a constant effective volume), without any additional biomass in the feed and neglecting the effect of gas exchanges. The time varying equations resulting from mass balances are given by [50]:

$$
\left\{\begin{array}{l}
\dot{X}(t)=\mu(Q(t), I(t)) X(t)-D X(t) \\
\dot{Q}(t)=\rho(S(t))-\mu(Q(t), I(t)) Q(t) \\
\dot{S}(t)=\left(S_{i n}-S(t)\right) D-\rho(S(t)) X(t)
\end{array}\right.
$$

where

- $D$ represents the dilution rate $\left(\mathrm{d}^{-1}, \mathrm{~d}\right.$ : day) which is the ratio of the inlet flow rate to the volume of the culture.

- $S_{\text {in }}$ the input substrate concentration $\left(\mu \mathrm{mol} \mathrm{L}^{-1}\right)$.

The specific uptake rate $\rho(S)$ is given by Monod kinetics:

$$
\rho(S)=\rho_{m} \frac{S}{S+K_{S}}
$$

The parameters $K_{s}$ and $\rho_{m}$ represent respectively the substrate half saturation constant and the maximal specific uptake rate.

The specific growth rate $\mu(Q, I)$, on the other hand, can be defined as a function of the internal quota $Q$ as follows (Droop model):

$$
\mu(Q, I)=\bar{\mu}\left(1-\frac{K_{Q}}{Q}\right) \mu_{I}(I)
$$

The theoretical maximal specific growth rate is denoted $\bar{\mu}$ and $K_{Q}$ represents the minimal cell quota, for which no algal growth can take place.

The light intensity has a direct effect on growth (photosynthesis), while uptake can continue in the dark. The modeling of the light effect consists in including the term $\mu_{I}$ in (70) which is represented by a Haldane type kinetics to model the photoinhibition [53]:

$$
\mu_{I}(I)=\frac{I}{I+K_{S I}+\frac{I^{2}}{K_{i I}}}
$$

where $I$ is the light intensity $\left(\mu \mathrm{E} \mathrm{m}^{-2} \mathrm{~s}^{-1}\right)$ and $K_{S I}$ and $K_{i I}$ are light saturation and inhibition constants respectively. The optimal light intensity that maximizes the function $\mu_{I}$ is given by $I_{\text {opt }}=\sqrt{K_{s I} K_{i I}}$. In the sequel, the light intensity is either set at this optimal value $I_{\text {opt }}$ or is time varying.

The parameters of the model used in this study are given in Table I [47],[54].

The nonlinear model (68) is represented thereafter in the state-space formalism (1) as follows:

$$
\left\{\begin{array}{l}
\dot{x}(t)=F(x, u, w, \theta), x\left(t_{0}\right)=x_{0} \\
y=X
\end{array}\right.
$$


Table I. Droop model parameters.

\begin{tabular}{ccc}
\hline Parameter & Value & Unit \\
\hline $\bar{\mu}$ & 2 & $\mathrm{~d}^{-1}$ \\
$\rho_{m}$ & 9.3 & $\mu \mathrm{mol} \mu \mathrm{m}^{-3} \mathrm{~d}^{-1}$ \\
$K_{Q}$ & 1.8 & $\mu{\mathrm{mol} \mu \mathrm{m}^{-3}}$ \\
$K_{s}$ & 0.105 & $\mu \mathrm{mol} \mathrm{L}^{-1}$ \\
$K_{s I}$ & 150 & $\mu \mathrm{E} \mathrm{m}^{-2} \mathrm{~s}^{-1}$ \\
$K_{i I}$ & 2000 & $\mu \mathrm{E} \mathrm{m}^{-2} \mathrm{~s}^{-1}$ \\
\hline
\end{tabular}

with

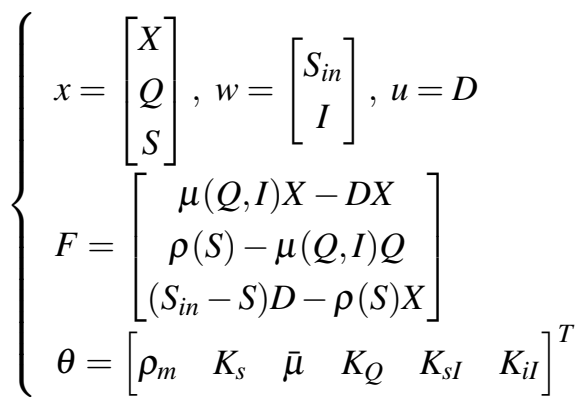

where the state variables are assembled in a vector denoted $x$ with $x_{0}$ its initial value. The nonlinear process dynamics are denoted $F$. The measurements are related to vector $y$ whereas the inputs are represented by vector $u$. The other exogenous inputs are denoted $w$. Finally, the parameters refer to vector $\theta$.

The steady states of the system are derived from three nonlinear equations, given in order to cancel out the model's dynamic equations, i.e.

$$
F\left(x_{e}, u_{e}, w, \theta\right)=0
$$

where $w$ is assumed constant and known. For a given value of $X$, the goal here is to characterize the corresponding values for $Q, S$ and $D$ for a constant light intensity $I$. Then, the equilibrium points are defined as follows (from (68)):

$$
\left\{\begin{array}{l}
\mu\left(Q_{e}, I\right)-D_{e}=0 \\
\rho\left(S_{e}\right)-\mu\left(Q_{e}, I\right) Q_{e}=0 \\
\left(S_{\text {in }}-S_{e}\right) D_{e}-\rho\left(S_{e}\right) X_{e}=0
\end{array}\right.
$$

Then, rearranging (75), the following system of equations has to be solved algebraically:

$$
\left\{\begin{array}{l}
D_{e}=\bar{\mu}\left(1-\frac{K_{Q}}{Q_{e}}\right) \mu_{I} \\
S_{e}=\frac{\mu\left(Q_{e}, I\right) Q_{e} K_{s}}{\rho_{m}-\mu\left(Q_{e}, I\right) Q_{e}} \\
\left(S_{i n}-\frac{\mu\left(Q_{e}, I\right) Q_{e} K_{s}}{\rho_{m}-\mu\left(Q_{e}, I\right) Q_{e}}\right)-Q_{e} X_{e}=0
\end{array}\right.
$$


Taking $Q_{e}$ as an unknown variable, the following quadratic equation must be solved:

$$
\begin{aligned}
& {\left[\bar{\mu} \mu_{I} X_{e}\right] Q_{e}^{2}-\left[\left(S_{i n}+K_{s}\right) \bar{\mu} \mu_{I}+\left(\rho_{m}+\bar{\mu} \mu_{I} K_{Q}\right) X_{e}\right] Q_{e}} \\
& +\left[\left(\rho_{m}+\bar{\mu} \mu_{I} K_{Q}\right) S_{i n}+\bar{\mu} \mu_{I} K_{Q} K_{s}\right]=0
\end{aligned}
$$

Leading to:

$$
Q_{e 1,2}^{*}=\frac{S_{i n}+K_{s}}{2 X_{e}}+\frac{1}{2} Q_{l}\left(1 \mp \sqrt{1+\alpha \frac{1}{X_{e}}+\beta \frac{1}{X_{e}^{2}}}\right)
$$

For each value $X_{e}$, there are two possible real solutions $Q_{e 1,2}^{*}$ given by (78). It can be shown that only one is feasible for the operating conditions considered in the sequel.

\subsection{Control law objectives}

The main objective of the controller is to regulate the biomass concentration $X$ to a reference value $X^{r}$ in the presence of parameter uncertainties and noise measurement, while the dilution rate $D$ is constrained to track the reference $D^{r}$ where $0 \leq D \leq D_{\max }$ ( $D_{\max }$ is the maximal dilution rate).

The NMPC problem is then formulated as:

$$
\min _{0 \leq D_{k}^{k+N_{p}-1} \leq D_{\max }}\left\|D_{k}^{k+N_{p}-1}-D_{k}^{r, k+N_{p}-1}\right\|_{V}^{2}+\left\|X_{k+1}^{k+N_{p}}-X_{k+1}^{r, k+N_{p}}\right\|_{W}^{2}
$$

and the RNMPC by:

$$
\min _{0 \leq D_{k}^{k+N_{p}-1} \leq D_{\max }} \max _{\theta \in\left[\theta^{-}, \theta^{+}\right]}\left\|D_{k}^{k+N_{p}-1}-D_{k}^{r, k+N_{p}-1}\right\|_{V}^{2}+\left\|X_{k+1}^{k+N_{p}}-X_{k+1}^{r, k+N_{p}}\right\|_{W}^{2}
$$

where the uncertain parameter subspace $\left[\theta^{-}, \theta^{+}\right]$is given by $\left[0.7 \theta_{\text {nom }}, 1.3 \theta_{\text {nom }}\right]$ with $\theta_{\text {nom }}$ given in Table I. This $30 \%$ mismatch has been chosen as a rather classic percentage in the literature. A more rigourous approach could be to proceed with an identification procedure as in [55] to determine the confidence intervals for all parameters.

The performance of the controllers in the case of disturbances (here the light intensity fluctuations) will be also studied and evaluated.

The efficiency of the proposed control strategies is validated in simulation. The initial biomass concentration value is set close to the setpoint in order to cancel the transient effect and focus only on the behavior during setpoint changes (rising and falling edge respectively). The light intensity is assumed to be non-measured, non-corrupted with noise. First, it is constant, equal to $I_{o p t}$ that maximizes $\mu(Q, I)$ defined in (70). Secondly, it is time-varying. The performance of the predictive algorithms is compared in a worst uncertain parameters case. Thanks to the monotonocity properties of the Droop model as discussed in [47], the worst-case prediction can be generated using parameter bounds $\left\{\theta^{-}, \theta^{+}\right\}$only, rather than by exploring the full parameter space $\left[\theta^{-}, \theta^{+}\right]$. The parameter values of the system are chosen on the parameter subspace border $\left(\theta_{\text {real }}=\left[\rho_{m}^{+}, K_{s}^{-}, \bar{\mu}^{+}, K_{Q}^{-}, K_{s I}^{-}, K_{i I}^{+}\right]\right)$. All the simulation conditions are summarized in Table II.

Two configurations may be considered for the determination of the pair $\left(D^{r}, X^{r}\right)$ of reference signals: 
Table II. Simulation conditions for the Droop model.

\begin{tabular}{llll}
\hline & Variable & Value & Unit \\
\hline sampling time & $T_{s}$ & 10 & $\mathrm{~min}$ \\
integration time step & $T_{d}$ & 12 & $\mathrm{sec}$ \\
simulation time & $T_{f}$ & 1 & $\mathrm{~d}$ \\
inlet substrate concentration & $S_{\text {in }}$ & 100 & $\mu \mathrm{mol} \mathrm{L}^{-1}$ \\
optimal light intensity & $I_{o p t}$ & 547 & $\mu \mathrm{E} \mathrm{m}^{-2} \mathrm{~s}^{-1}$ \\
maximal cell quota & $Q_{l}$ & 9 & $\mu \mathrm{mol}^{-3}$ \\
maximal admissible dilution rate & $D_{\max }$ & 1.6 & $\mathrm{~d}^{-1}$ \\
prediction horizon & $N_{p}$ & 5 & - \\
weighting matrix on control & $V$ & $\square_{N_{p}}$ & - \\
weighting matrix on state & $W$ & $\square_{N_{p}}$ & - \\
initial biomass concentration & $X(0)$ & 24.95 & $\mu \mathrm{m}^{3} \mathrm{~L}^{-1}$ \\
initial internal quota & $Q(0)$ & 4 & $\mu \mathrm{mol} \mathrm{m}^{-3}$ \\
initial substrate concentration & $S(0)$ & 0.05 & $\mu \mathrm{mol} \mathrm{L}^{-1}$ \\
\hline
\end{tabular}

- Case 1: $D^{r} \longrightarrow X^{r}$

The biomass reference trajectory $X^{r}$ is obtained by applying the dilution rate reference trajectory $D^{r}$ to the model.

- Case 2: $X^{r} \longrightarrow D^{r}$

In case of constant $X^{r}$ (with an assumed constant light intensity), the dilution rate reference trajectory $D^{r}$ is computed from the knowledge of the target setpoint $X^{r}$ using relations at equilibrium (76):

$$
D^{r}=\bar{\mu}\left(1-\frac{K_{Q}}{Q^{r}}\right) \mu_{I}
$$

with $Q^{r}$ the reference internal quota given by (78).

In case of time-varying $X^{r}$ or $X^{r}$ constant with a time-varying light intensity, the dilution rate reference trajectory $D^{r}$ could be determined by solving the following constrained open-loop optimization problem:

$$
D^{r}(t)=\arg \min _{0 \leq D(t) \leq D_{\max }}\left|X(t)-X^{r}(t)\right|^{2}
$$

\subsection{Numerical results}

6.3.1. Constant light intensity The performance of setpoint tracking is first studied. The goal is to track the biomass concentration setpoint (rising and falling step changes as illustrated in Figure 1), corresponding to case 1. Three predictive control laws are tested (Figures 1 and 2): a classic Nonlinear Model Predictive Control (denoted as NMPC), a robust NMPC (denoted as RNMPC) and the linearized one (LRMPC). Biomass concentration measurements, $y_{k}$, are assumed to be corrupted by centred Gaussian white noise with 0.1 standard deviation.

The anticipation behavior to a setpoint change (Figure 1) for all controllers can be noted thanks to the prediction of the future evolution of the setpoint trajectory over the moving horizon. The dilution rate decrease leads to an increase in the cell concentration (for example between $0.2 \mathrm{~d}$ and $0.3 \mathrm{~d}$ in Figure 2) and vice versa (for example between $0.7 \mathrm{~d}$ and $0.8 \mathrm{~d}$ ), which agrees with the 


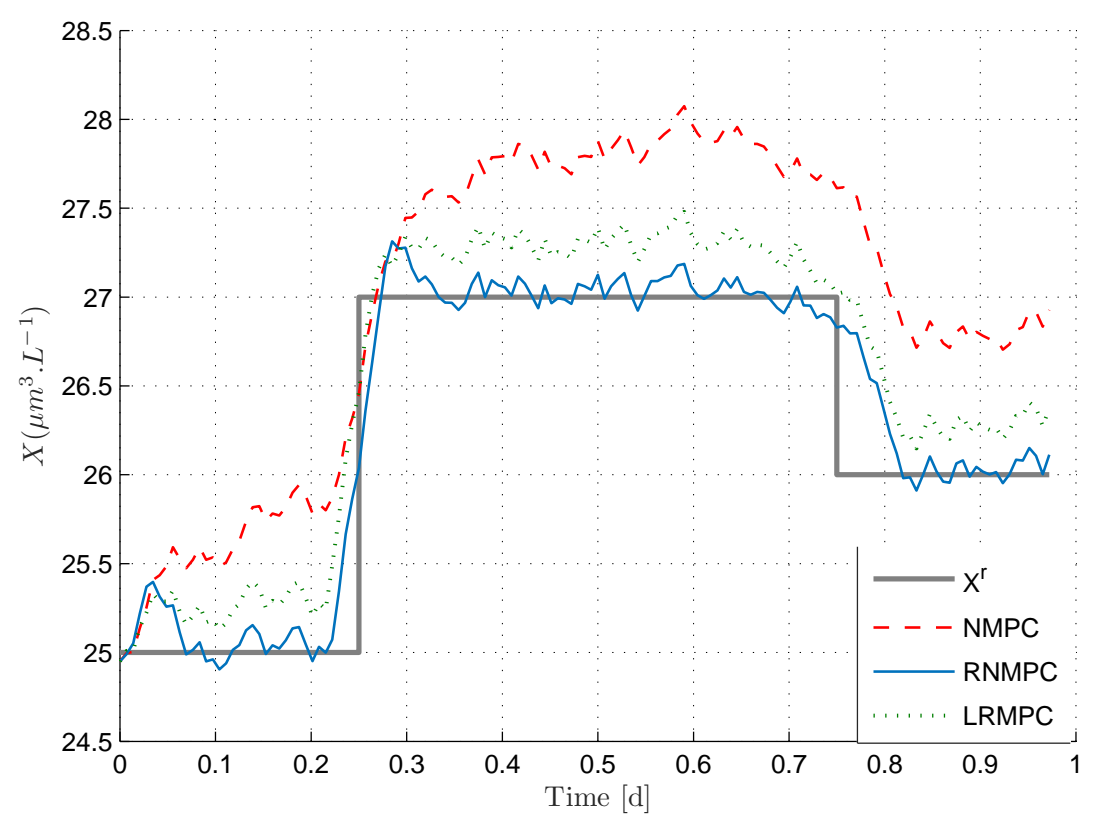

Figure 1. Biomass concentration evolution over time for NMPC, RNMPC and LRMPC strategies.

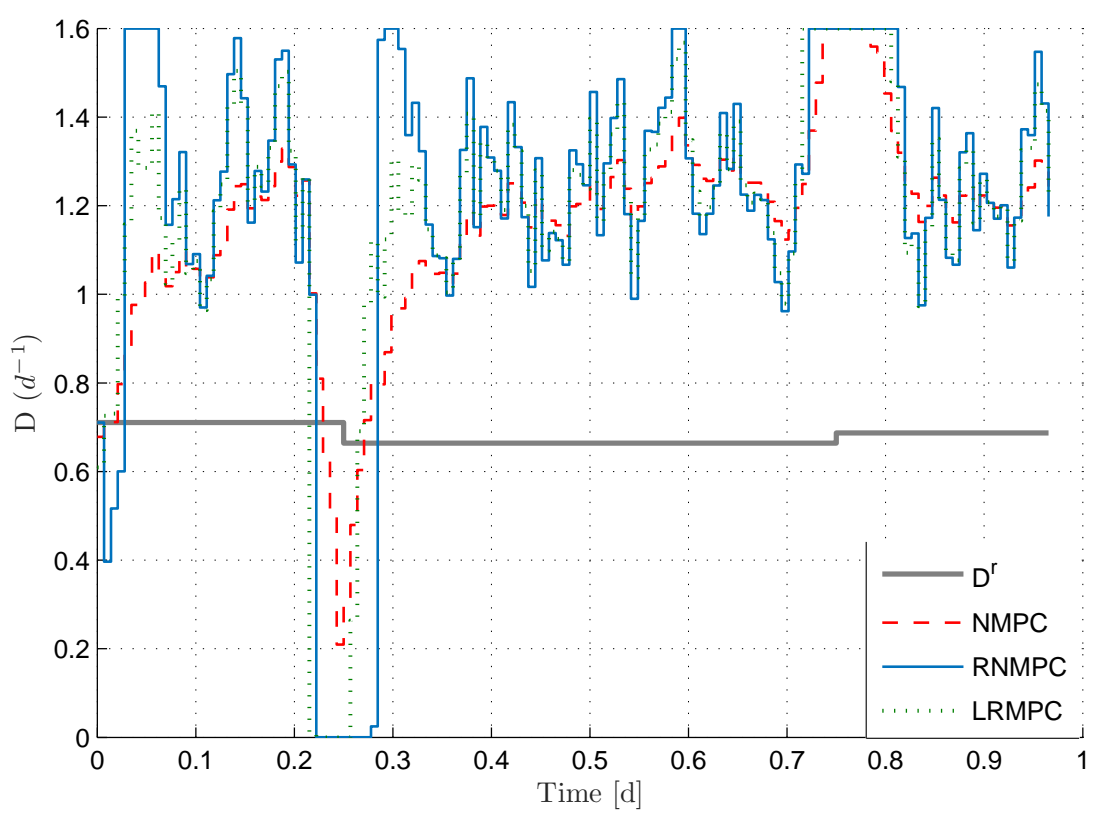

Figure 2. Dilution rate evolution over time for NMPC, RNMPC and LRMPC strategies.

biological aspect. The obtained results, as expected, show that both RNMPC and LRMPC perform better than the classic NMPC under parameter uncertainties and measurement noise. In the NMPC law, the biomass concentration is not able to track the specified setpoint in the presence of parameter uncertainties, due to the fact that the mismatch between the system and the model is not considered during the prediction step inside the minimization procedure. Furthermore, the RNMPC performs better than the LRMPC controller under parameter uncertainties in terms of tracking accuracy but the computational burden of the former is much higher. The LRMPC algorithm performs well and 
offers a very significant computational load reduction compared with RNMPC as shown in Table III. In fact, this can be explained by the fact that RNMPC is an optimization problem of dimension $N_{p} \times n_{u} \times n_{\theta}$ while LRMPC is a bilevel optimization problem. Consequently, when considering a more complex model with a greater number of state variables and parameters, the computation time increases quickly in the RNMPC strategy, becoming much higher than the sampling rate.

Table III. Comparison of the predictive algorithms in terms of computation time at each sampling time.

\begin{tabular}{|cc|c|c|c|}
\cline { 4 - 5 } \multicolumn{2}{c|}{} & \multicolumn{3}{|c|}{ Computation time (s) } \\
\hline \multirow{2}{*}{ Algorithm } & & $\min$ & $\operatorname{mean}$ & $\max$ \\
\hline & NMPC & $<10^{-5}$ & 0.014 & 0.29 \\
& RNMPC & 0.55 & 1.85 & 19.31 \\
LRMPC & $<10^{-5}$ & 0.01 & 0.09 \\
\hline
\end{tabular}

The performance of the LRMPC law can even be improved when reducing sampling time $T_{S}$ in order to guarantee that the first order Taylor series expansion is as accurate as possible, leading to a reduced steady state error [56].

A statistical analysis of the robustness is considered in order to highlight the advantage of the proposed approach. To emphasize this aspect, simulation for a large number of independent tests is performed with parameter variations for the same conditions as previously. Based on the Monte-Carlo procedure, 100 tests have been conducted with a simultaneous random non-correlated variation of $30 \%$ at the maximum in all the parameters $\theta$. Figure 3 compares NMPC and LRMPC performance. It clearly appears that using the LRMPC algorithm (solid line in blue) makes it possible to reduce the standard deviation of the tracking error in comparison with a classic NMPC (dotted line in red).

Figure 4 shows the resulting histogram of the distribution of the tracking error, and Table IV gives the mean and standard deviation of the tracking error for the whole simulation.

Table IV. Comparison of the proposed algorithms in terms of tracking error distribution features.

\begin{tabular}{|cc|c|c|}
\hline Algorithm & Performance indices & mean & standard deviation \\
\hline \multicolumn{2}{|c|}{} & & \\
\hline & NMPC & -0.07 & 0.219 \\
& LRMPC & -0.05 & 0.014 \\
\hline
\end{tabular}

These results confirm those obtained in the case depicted in Figure 1. Furthermore, Figure 4 shows that the LRMPC has better results than the NMPC in terms of accuracy.

6.3.2. Variation of the light intensity The light intensity was set constant in the previous simulations (equal to $I_{o p t}$ ). In this section, the behavior of the controller in case the of time-varying light intensity is discussed. Hereafter, a day/night-like variation is considered [57]:

$$
I_{\text {real }}(t)=\bar{I}(\max \{0, \sin (2 \pi t)\})^{2}+I_{o p t}
$$




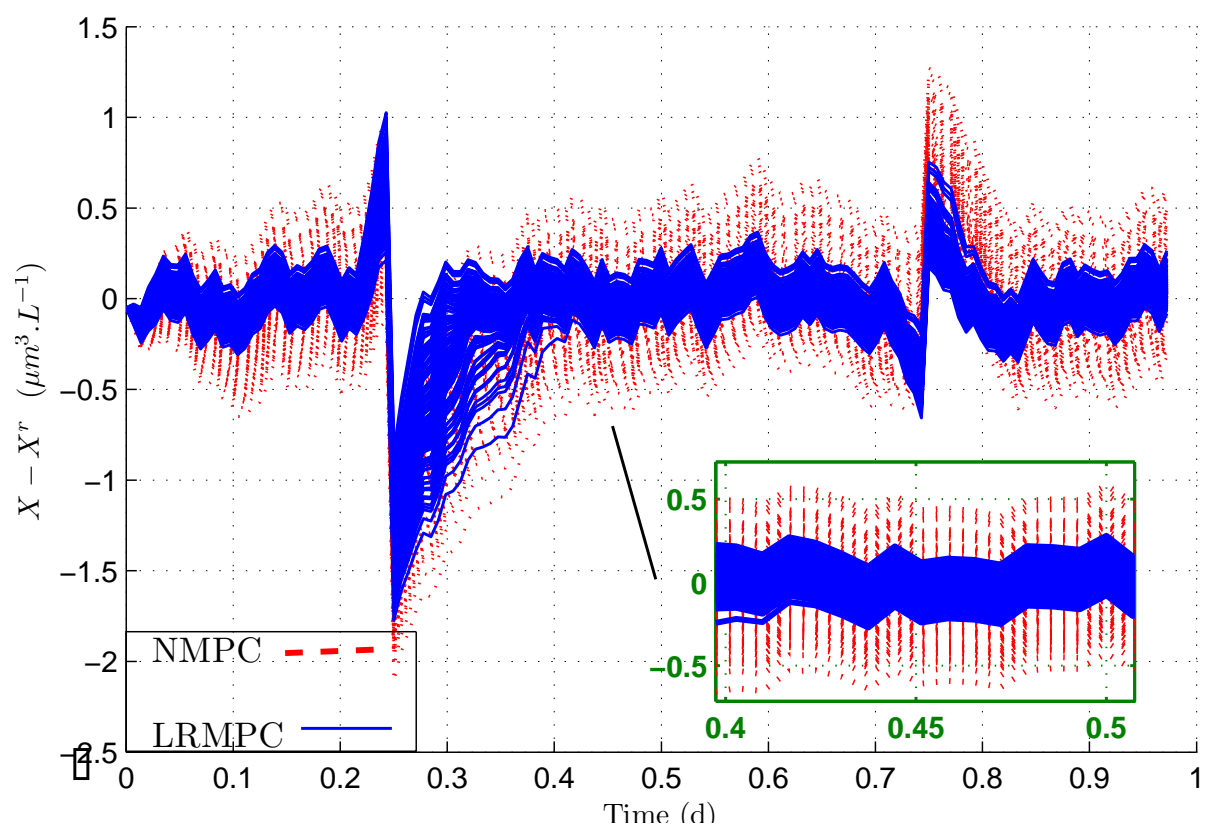

Figure 3. Time evolution of tracking error for simultaneous random non-correlated variations in all the parameters (Monte-Carlo).

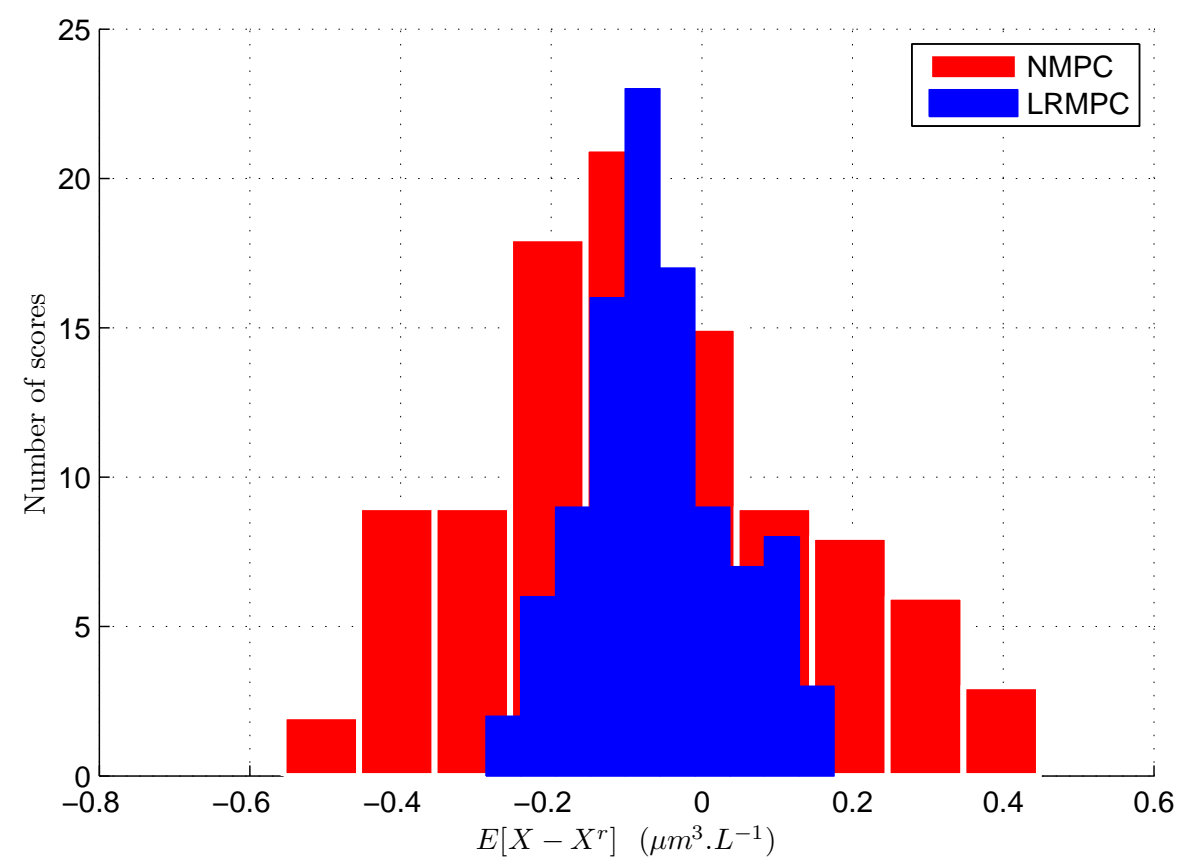

Figure 4. Histogram of the average tracking error for simultaneous random non-correlated variations in all the parameters (Monte-Carlo).

where the time $t$ is in days and $\bar{I}$ is set to $280 \mu \mathrm{E} \mathrm{m}^{-2} \mathrm{~s}^{-1}$. $I_{\text {nominal }}=I_{\text {opt }}$ represents the light energy provided by panels to the bioreactor and $I_{\text {real }}$ the perturbation modelled as a day/night cycle (i.e. non-perfectly isolated culture) as shown in Figure 5.

The biomass concentration setpoint is constant $\left(X^{r}=25 \mu \mathrm{m}^{3} / L\right)$. The goal is thus to maintain the 


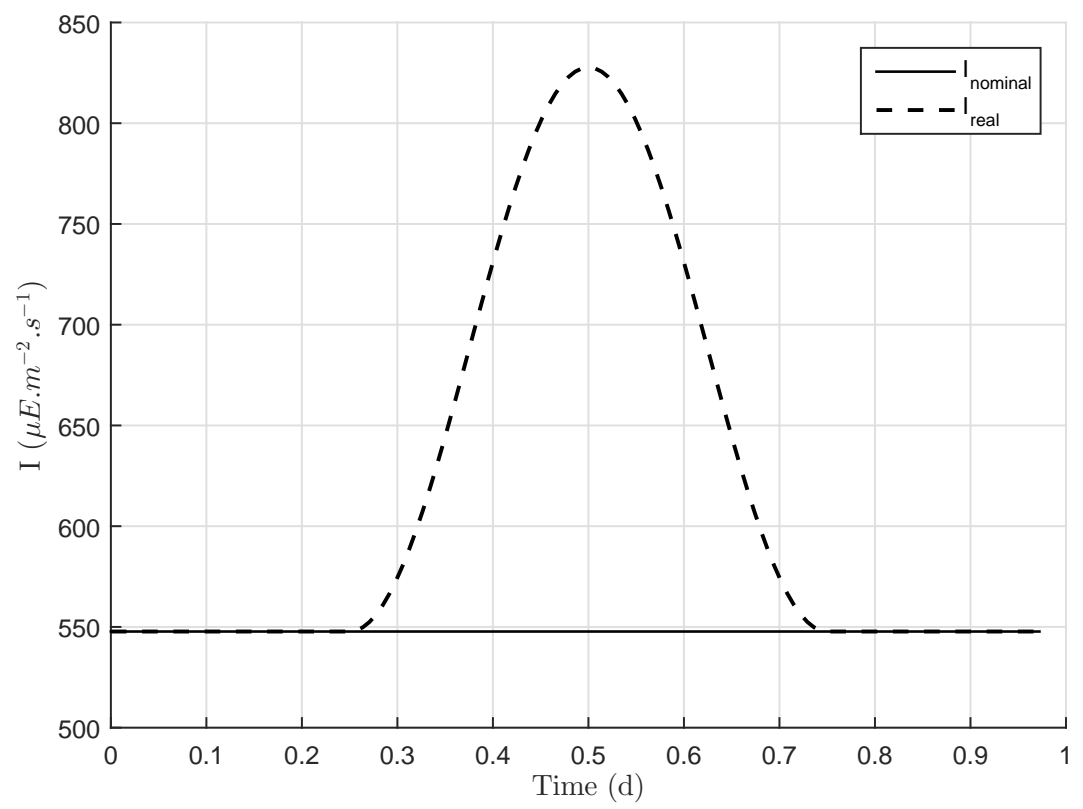

Figure 5. Light intensity evolution over time.

biomass concentration as close as possible to this value, despite the light intensity fluctuations. Figures 6-7 compare the NMPC, RNMPC and LRMPC controller performance. The light intensity considered in the model for prediction is $I_{\text {nominal }}$.

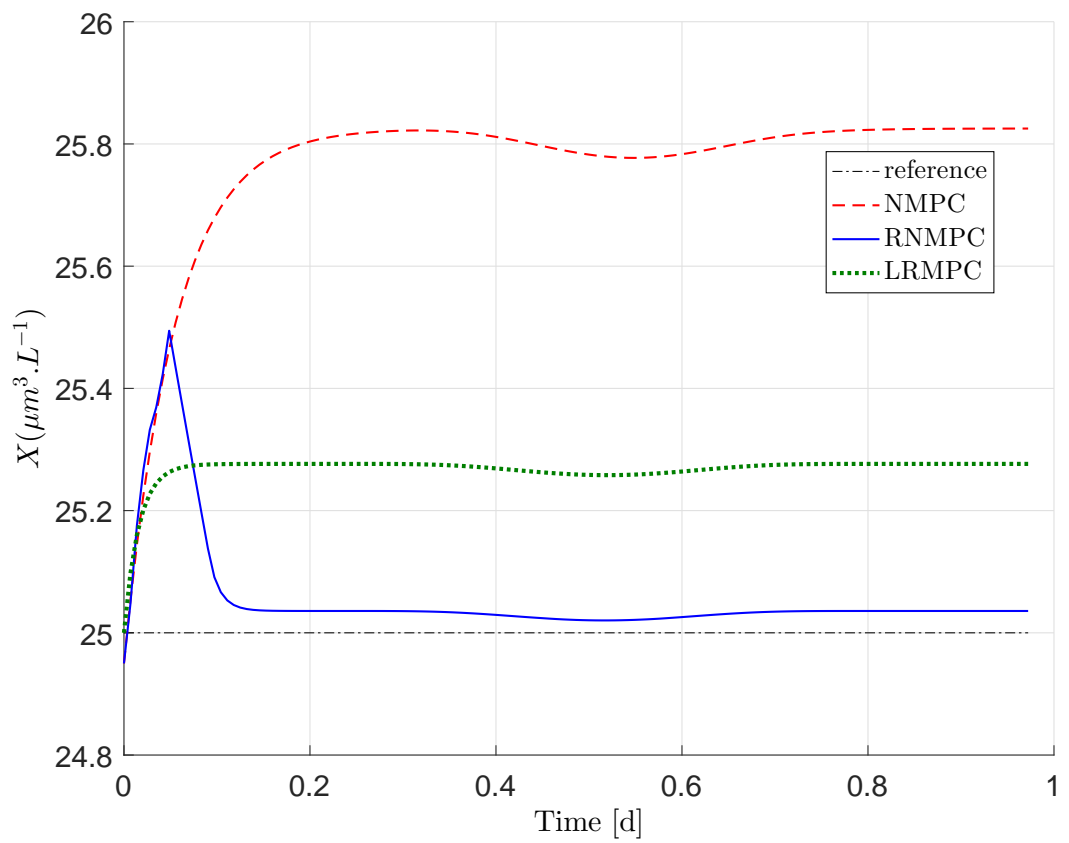

Figure 6. Biomass concentration evolution over time for NMPC, RNMPC and LRMPC strategies. 


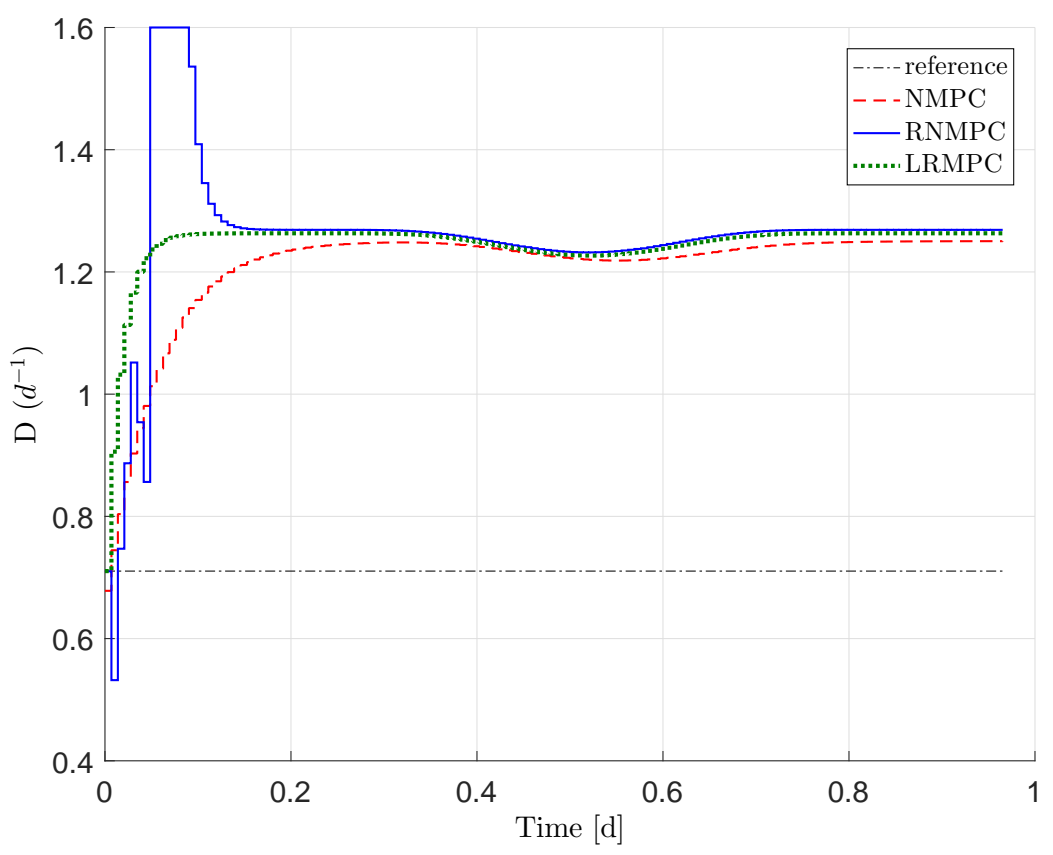

Figure 7. Control input evolution over time for NMPC, RNMPC and LRMPC strategies.

A steady tracking error can be observed for all the controllers. The NMPC law leads to the highest error (about $0.8 \mu \mathrm{m}^{3} \mathrm{~L}^{-1}$ ), whereas the RNMPC strategy leads to the best result. The developed LRMPC leads to a tracking error about $0.3 \mu \mathrm{m}^{3} \mathrm{~L}^{-1}$. Indeed, since the LRMPC approaches the RMPC problem, it leads to a slight loss in performance, but with a reduction in computation time. It can be noticed that for all controllers, the impact of light variations on the biomass concentration is well reduced by decreasing the dilution rate according to growth rate fluctuations (due to light intensity variations), so that the biomass concentration is maintained constant at its reference value. 


\section{CONCLUSIONS AND FUTURE WORK}

In this paper, the robustification of a nonlinear model predictive controller in the case of model uncertainties was studied. Considering a process model with parameters that are within given confidence intervals, the min-max robust MPC can be designed in order to take account of these parameter uncertainties. The optimal control sequence is then determined so that the maximum deviation for all trajectories over all possible data scenarii is minimized. In this paper, a new linearized robust NMPC strategy is presented. The goal is to turn the min-max optimization problem involved in the RNMPC algorithm into a more tractable one. The proposed strategy is based on the linearization of the predicted trajectory over the reference control sequence and the nominal parameter values. The obtained optimization problem is a bilevel one: a unidimensional optimization problem in the upper level, and a QP problem in the lower one. Several simulations were performed in order to compare the proposed LRMPC strategy to the min-max RNMPC in the case of model parameter uncertainties and disturbances in the case of a microalgae cultivation system. The LRMPC ensures a good trade-off between computational load and tracking trajectory accuracy.

Future research will focus on the impact of the convergence and feasability of the optimization algorithm on the stability and performance of the control law.

One interesting perspective in order to cancel any residual tracking error may be to consider a hierarchical control scheme that combines a robust model predictive control law with a proportional Integral (PI) law.

Handling unstructured uncertainties by the controller should also be investigated. Moreover, the biological variables are sometimes not accessible for measurement online and are usually measured offline using expensive sensors. In this context, it is important to design an estimation algorithm or so-called soft sensors to rebuild the time evolution of the state and develop a constructive procedure for designing controllers robust in the face of additional estimation errors. Contributions in this area are particularly important.

\section{REFERENCES}

1. Robust and nonlinear control literature survey (No. 9). International Journal of Robust and Nonlinear Control 2008. John Wiley \& Sons, Ltd; 18(18):1681-1685.

2. Pepe P, Di Ciccio MP. ISS feedback control redesign for continuous stirred tank reactors. International Journal of Robust and Nonlinear Control 2011. John Wiley \& Sons, Ltd; 21(17):1947-1974.

3. Rehman OU, Petersen IR, Pota H. Nonlinear robust state feedback control system design for nonlinear uncertain systems. International Journal of Robust and Nonlinear Control 2016. John Wiley \& Sons, Ltd

4. Mayne DQ, Rakovi, S. Model predictive control of constrained piecewise affine discrete-time systems. International Journal of Robust and Nonlinear Control 2003. John Wiley \& Sons, Ltd; 13(3-4):261-279.

5. Dubljevic S, El-Farra NH, Mhaskar P, Christofides PD. Predictive control of parabolic PDEs with state and control constraints. International Journal of Robust and Nonlinear Control 2006. John Wiley \& Sons, Ltd; 16(16):749-772.

6. Camacho EF, Bordons C. Model Predictive Control. Springer London 2004.

7. Maciejowski JM. Predictive Control: With Constraints. Prentice Hall 2002.

8. Mishra SK. Topics in Nonconvex Optimization: Theory and Applications. Springer Optimization and Its Applications 2011.

9. Lelić MA, Zarrop MB. Generalized pole-placement self-tuning controller Part 1, Basic algorithm. International Journal of Control 1987; 46(2):547-568. 
10. Linkers DA, Mahfonf M. Advances in Model-Based Predictive Control. Oxford University Press 1994; Ch. Generalized Predictive Control in Clinical Anesthesia.

11. Sánchez JMM, Rodellar J. Adaptive Predictive Control: From the Concepts to Plant Optimization. Prentice-Hall international series in systems and control engineering 1996.

12. Clarke DW. Application of generalized predictive control to industrial processes. IEEE Control Systems Magazine 1988; 8(2):49-55.

13. Huang R, Zavala VM, Biegler LT. Advanced step nonlinear model predictive control for air separation units. Journal of Process Control 2009; 19(4):678 - 685.

14. Richalet J. Industrial applications of model based predictive control. Automatica 1993; 29(5):1251 - 1274.

15. Richalet J, Rault A, Testud JL, Papon J. Model predictive heuristic control: Application to industrial processes. Automatica 1978; 14(2):413-428.

16. Harinath E, Biegler LT, Dumont GA. Control and optimization strategies for thermo-mechanical pulping processes: Nonlinear model predictive control. Journal of Process Control 2011; 21(4):519 - 528.

17. Nagy ZK, Braatz, RD. Robust nonlinear model predictive contro of fedbatches processes. AIChE Journal 2009; 49(7):1776-1786.

18. Ashoori A, Moshiri B, Khaki-Sedigh A, Bakhtiari MR. Optimal control of a nonlinear fed-batch fermentation process using model predictive approach. Journal of Process Control 2009; 9:1162-1173.

19. Tebbani S, Lopes F, Filali R, Dumur D, Pareau D. Nonlinear predictive control for maximization of $\mathrm{CO}_{2}$ bio-fixation by microalgae in a photobioreactor. Bioprocess and Biosystems Engineering 2014; 37:83-97.

20. Tebbani S, Dumur D, Hafidi G, Vande Wouwer A. Nonlinear Predictive Control of fed-batch Cultures of E. coli. Chemical Engineering \& Technology 2010; 33:1112-1124.

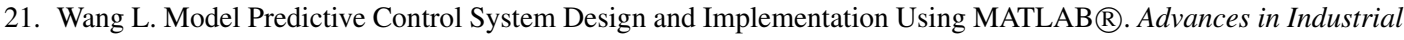
Control 2009.

22. García CE, Prett DM, Morari M. Model predictive control: Theory and practice-A survey. Automatica 1989; 25(3):335 - 348.

23. Hartley EN, Jerez JL, Suardi A, Maciejowski JM, Kerrigan EC, Constantinides GA. Predictive Control Using an FPGA With Application to Aircraft Control. IEEE Transactions on Control Systems Technology 2014; 22(3):10061017.

24. Bourgeois E, Tebbani S, Ramos Espinosa A. Launcher atmospheric guidance based on nonlinear model predictive control. GNC 2014

25. Crassidis J, Markley F, Anthony TC, Andrews S. Nonlinear predictive control of spacecraft. Journal of Guidance, Control, and Dynamics 1997; 20(6):1096-1103.

26. Re LD, Allgöwer F, Glielmo L, Guardiola C, Kolmanovsky I. Automotive Model Predictive Control: Models, Methods and Applications. Springer London 2010.

27. Balchen JG, Ljungquist D, Strand S. State Space Model Predictive Control of a Multi Stage Electro-metallurgical Process. Modeling, Identification and Control 1989; 10(1):35-51.

28. Nguyen XM, Lawayeb F, Rodriguez-Ayerbe P, Dumur D, Mouchette A. Nonlinear model predictive control of steel slab walking-beam reheating furnace based on a numerical model. Proceeding of the IEEE Multi-Conference on Systems and Control 2014:191-196.

29. Cannon M, Ng D, Kouvaritakis B. Nonlinear Model Predictive Control: Towards New Challenging Applications, chapter Successive Linearization NMPC for a Class of Stochastic Nonlinear Systems. Springer Berlin Heidelberg 2009:249-262.

30. Limon D, Alamo T, Salas F, Camacho EF. Input to state stability of min-max MPC controllers for nonlinear systems with bounded uncertainties. Automatica 2006; 42(5):797-803.

31. Magni L, De Nicolao G, Scattolini R, Allgöwer F. Robust model predictive control for nonlinear discrete time systems. International Journal of Robust and Nonlinear Control 2003; 13:229-246.

32. Magni L, Scattolini R. Assessment and Future Directions of Nonlinear Model Predictive Control, chapter Robustness and Robust Design of MPC for Nonlinear Discrete-Time Systems. Springer Berlin Heidelberg 2007:239-254.

33. Michalska H, Mayne DQ. Robust receding horizon control of constrained nonlinear systems. IEEE Transactions on Automatic Control 1993; 38(11):1623-1633.

34. Kerrigan EC, Maciejowski J. Feedback min-max model predictive control using a single linear program: Robust stability and the explicit solution. International Journal of Robust Nonlinear Control 2004; 14:395-413.

35. Limon D, Alamo T, Camacho EF. Robust stability of min-max MPC controllers for nonlinear systems with bounded uncertainties. Proceeding of the mathematical Theory of Networks and Systems 2004.

36. Magni L, Scattolini R. Robustness and robust design of MPC for nonlinear discrete-time systems. Springer-Verlag 2007; 358:239-254. 
37. Mayne DQ. Control of Constrained Dynamic Systems. European Journal of Control 2001; 7:87-99.

38. Raimondo DM, Limon D, Lazar M, Magni L, Camachp EF. Min-max Model Predictive Control of Nonlinear Systems: A Unifying Overview on Stability. European Journal of Control 2009; 5:5-21.

39. Raimondo DM, Limon D, Alamo T, Magni L. Robust Model Predictive Control Algorithms for Nonlinear Systems: An Input-to-State Stability Approach. Model Predictive Control 2010.

40. Mayne DQ, Rawlings JB, Rao CV, Scokaert POM. Constrained model predictive control: Stability and optimality. Automatica 2000; 36:789-814.

41. Biegler LT, Yang X, Fischer GAG. Advances in sensitivity-based nonlinear model predictive control and dynamic real time optimization. Journal of Process Control 2015; 30:104-116.

42. Scokaert POM, Mayne DQ, Rawlings JB. Suboptimal model predictive control (feasibility implies stability. IEEE Transactions on Automatic Control 1999; 44(3):648-654.

43. Allwright JC. Advances in Model-Based Predictive Control. Oxford University Press 1994; Ch. On min-max Model-Based Predictive Control.

44. Magni L, Scattolini R. Assessment and Future Directions of Nonlinear Model Predictive Control. Springer Berlin Heidelberg 2007; Ch. Robustness and Robust Design of MPC for Nonlinear Discrete-Time Systems:239-254.

45. Du DZ, Pardalos PM. Minimax and Applications. Springer US 2013; Nonconvex Optimization and Its Applications.

46. Kasperski A. Discrete Optimization with Interval Data: Minmax Regret and Fuzzy Approach. Springer Berlin Heidelberg 2008; Studies in Fuzziness and Soft Computing.

47. Goffaux G, Vande Wouwer A. Design of a Robust Nonlinear Receding-Horizon Observer-Application to a biological system. Proceeding of the 17th IFAC World Congress 2008; 15553-15558.

48. Sayed AH, Nascimento VH, Cipparrone FAM. A Regularized Robust Design Criterion for Uncertain Data. SIAM Journal of Mathematical Analysis and Applications 2002; 32(4):1120-1142.

49. Boyd SP, Vandenberghe L. Convex Optimization. Cambridge University Press 2004.

50. Masci P, Grognard F, Bernard O. Microalgal biomass surface productivity optimization based on a photobioreactor model. 11th IFAC Symposium on Computer Applications in Biotechnology 2010; 180-185.

51. Bernard O. Hurdles and challenges for modelling and control of microalgae for $\mathrm{CO}_{2}$ mitigation and biofuel production. Journal of Process Control 2011; 21(10):1378-1389.

52. Bernard O, Gouzé J-L. Transient Behavior of Biological Loop Models, with Application to the Droop Model. Mathematical Biosciences 1995; 127(1):180-185.

53. Peeters JCH, Eilers PHC. The relationship between light intensity and photosynthesis. Hydrobiological Bulletin 1978; 12:134-136.

54. Munoz-Tamayo R, Martinon P, Bougaran G, Mairet F, Bernard O. Getting the most out of it: optimal experiments for parameter estimation of microalgae growth models. Journal of Process Control 2014; 24(6):991-1001.

55. Breitenecker F, Kugi A, Troch I, Benavides M, Telen D, Lauwers J, Logist F, Van Impe J, Vande Wouwer A. Parameter Identification of the Droop Model using Optimal Experiment Design. IFAC-PapersOnLine 8th Vienna International Conference on Mathematical Modelling 2015; 48(1):586-591.

56. Benattia SE, Tebbani S, Dumur D. A linearized robust model predictive control applied to bioprocess. Proceeding of the 55th IEEE Conference on Decision and Control 2016.

57. Tebbani S, Titica M, Join C, Fliess M, Dumur D. Model-based versus model-free control designs for improving microalgae growth in a closed photobioreactor: Some preliminary comparisons. Proceeding of the 24th Mediterranean Conference on Control and Automation 2016. 